\title{
BALANCING AN AIRCRAFT WITH SYMMETRICALLY DEFLECTED SPLIT ELEVATOR AND RUDDER DURING SHORT LANDING RUN
}

\author{
Mohammed BA ZUHAIR* \\ Department of Aerohydrodynamics, Faculty of Aviation, Land Transport and Energetics, Kazan National \\ Research Technical University - KAI, Republic of Tatarstan, 420097 Kazan, Russia
}

Received 11 July 2018; Accepted 23 April 2019

\begin{abstract}
This article investigates methods for balancing aircraft during short straight-line landing run realized by employing split rudder and elevator as air-brakes after touchdown. For standard atmospheric and runway conditions, directional and longitudinal balance equations for aircraft of conventional configuration such as Il-86 are presented. Methods depend on operational and mechanical approaches, where the first requires manual or automatic trim of shortly peaking small pitching, yawing, and rolling moments using dynamic forces while the second suggest some re-design of elevator and rudder control channels to limit deflection angles. The paper describes in detail each method disadvantages and suggests the adoption of automatic operational approach due to less required system modifications and piloting skills.
\end{abstract}

Keywords: air-brake, short landing run, take-off and landing performance, split elevator and rudder, two sectioned rudder, two sectioned elevator.

\section{Introduction}

Improvement of take-off and landing characteristics of wide-body transport aircraft remains one of the prioritized tasks whose innovative solution will increase profitability per flight. In general, particular interest is dedicated to shorten take-off and landing phases for achieving less fuel burn and noise as well as preserving urban resources by minimizing runway lengths and airport areas.

Short landing can be performed by implementing three methods: aerodynamic, mechanical, and thrust vectoring. Mechanical method utilizes landing gear braking system to utilize friction for aircraft deceleration, while thrust vectoring is using thrust reversers for some backward reaction force generation. Aerodynamic method as the most widespread is based on the full deployment of high-lift devices, namely, air-brakes and lift-dumpers in addition to various types of conventional and unconventional spoilers (Mertol, 2008). Besides, posterior/tailmounted and fuselage-mounted dorsal air-brakes were applied, for instance, in Buccaneer and passenger aircraft BAE-146 (Jung, 2012). The application of sectioned control surfaces for drag increase during landing mode were previously introduced and implemented. For example, chord-wise split elevons designed for B-2 are used for quick deceleration and trim (Jung, 2012). In addition, both
American and Russian Space Shuttles were equipped with vertically unfolded split-rudders (NASA, 2007) functioning as air-brakes during landing approach and run. Nevertheless, large-scale adoption of the posterior/tail-mounted air-brakes and foldable split-rudders as supplementary air-brakes have proven to be impractical for aircraft of conventional configuration because of control system complication and tail section overweighting. In addition, fuselage-mounted dorsal air-brakes require an unavailable installation volume and lead to increase fuselage drag during cruise flight (Mertol, 2008).

Multi-functionalization of the existing pitch and yaw sectioned control surfaces of wide-body long-haul aircraft such as A380-800, B747-400, B747-8i, and Il96-300 etc. to execute air-brake function can be an alternative promising solution for shortening landing run especially considering the current availability of its required system components, which will overcome the aforementioned disadvantages. Therefore, some future aircraft concepts similar to BWB (Liebeck, 2004; Ba Zuhair, 2018) are considering integration of split drag-rudders, split drag-elevons, and split drag-flaps with the design of future aircraft control systems.

In this context, a new aerodynamic method-based application is being developed to achieve short landing

*Corresponding author. E-mail: mohammed-ba@yandex.ru 
by symmetrical deflection of SR and SE sections up to $15-45^{\circ}$ after touchdown, a procedure synchronized with the deployment of high-lift devices and thrust reversers. Preliminary computational analysis within the framework of this research showed landing run shortening of 5.6\% when thrust reversers are on and $7.1 \%$ when the latter is off. During abnormal weather conditions, every increase of headwind speed by $1 \mathrm{~m} / \mathrm{s}$ may contribute further shortening of $2.5 \%$ (Bazuhair, 2018). As a conclusion, sectioning rudder and elevator was introduced as effective integrated air-brakes. However, untrimmed pitching, rolling, and yawing moments were discovered after full deflection of the SR and SE sections primarily created by the different arm lengths of aerodynamic forces at each section of the said control surfaces on the corresponding HS and VS.

In this work, all effort is devoted to investigate operational approaches capable of maintaining aircraft balance during a straight-line landing under standard weather conditions.

\section{CFD simulation and validation}

Geometric and aerodynamic inputs for the numerical study to solve the stability and trim mathematical equations were imported from the computational analysis of the Russian aircraft Il-86. Firstly, a three-dimensional model for of Il-86 with landing configuration was designed in SOLIDWORKS-2016 based on data from (Bekhtir, 1991), and further simulated using the built-in Flow Simulation package with k- $\varepsilon$ intensity and length turbulence model code (Alyamovsky, 2012). Flow Simulation is a software fully integrated in SOLIDWORKS for computing fluid

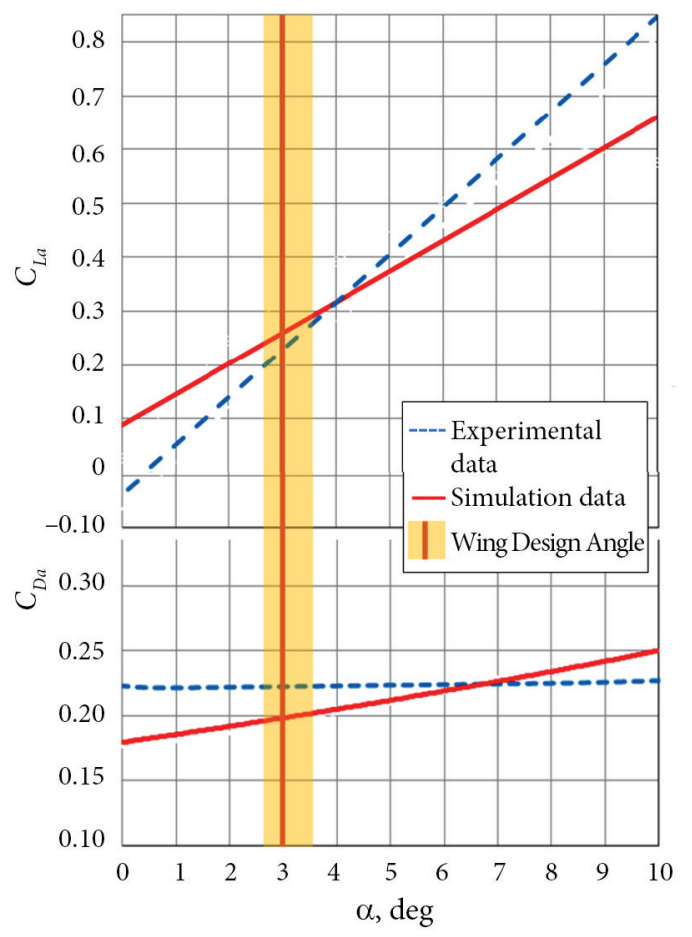

Figure 1. Validation graph of the computed $C_{D a}$ and $C_{L a}$ against experimental data provided by (Bekhtir, 1991) (gas or liquid) flows inside and outside SOLIDWORKS models, as well as heat transfer to (from, between, in) these models due to convection, radiation, and conduction with a proved CFD technology based on solving RANS equations (Dassualt-Systems, 2015). Structured mesh statistics, simulation settings and validation results were detailed in (Bazuhair, 2018). Related initial conditions include $V_{l}$ set at $77.87 \mathrm{~m} / \mathrm{s}$ and $\mathrm{Re}=40.6 \times 10^{6}$ in stable atmospheric medium and standard runway surface conditions recommended for numerical and experimental tests (Federal Aviation Administration [FAA], 2018). Validation graph of $C_{D \alpha}$ and $C_{L \alpha}$ obtained by the simulation and Il-86 test flights shows sufficiently valid results around the interested $\alpha_{d}=+3^{\circ}$ governing post-touchdown landing trajectory, see Figure 1.

\section{Numerical analysis and discussion}

Further analysis utilizes a mathematical model with geometrical and aerodynamic variables and constants indexed in accordance with the general form ( $\mathrm{j} . \mathrm{k}$ ) explained as follows: "j" stands for tail part, i.e. elevator (symbolized as - e.) or rudder (symbolized as - r.), while " $k$ " refers to the location of the attached control surface section to “ $j$ " with respect to the horizontal axis $O x$ of aircraft-body coordinate system, where: right/left $-r / l$, root/tip $-r / t$, and lower/upper - 1/u. For example, the index "r.rr" below $A_{\text {r.rr }}$ is read as: "area of the right root sections of SR", see notions for additional examples. Calculations are run assuming these design parameters and operational considerations:

1. Equal areas of $\mathrm{SE}$ and $\mathrm{SR}$ sections. Therefore, $A_{\text {e.rk }}=A_{\text {e.lk }}=0.25 A_{\mathrm{e}}$, and $A_{\mathrm{r} . \mathrm{u}}=A_{\mathrm{r} . \mathrm{l}}=0.5 A_{\mathrm{r}}$;

2. ymmetrical deflection angles $\delta_{j . k}$ of SE and SR sections. Accordingly, $\delta_{\text {r.l }}=-\delta_{\text {r.u }}$, similarly $\delta_{\text {e.kr }}=-\delta_{\text {e.kt }}$, where $\delta_{\text {r.u }}=\delta_{\text {e.kt }} \in\left[0^{\circ}, 45^{\circ}\right]$;

3. Landing is performed on flat concrete runway at standard atmospheric conditions with functional engines, i.e. zero-sideslip angle.

For analysis simplification, the investigated unsteady motion of aircraft is assumed to occur on three or more contact points with runway surface within a timeframe starting from touchdown to stop. Accordingly, aircraft may be observed as a rigid body with applied aerodynamic and inertial forces including $L_{\alpha}, D_{\alpha}, W_{l}$, and $T_{1 j, 2 j}$ with magnitude of nominal or reverse thrust mode values and relevant vector direction as well as $F_{n}$ and $F_{\text {r.k }}$ along with the relevant $N_{\mathrm{n}}$ and $N_{\text {r.k }}$. Additionally, aerodynamic forces $L_{j . \mathrm{k}}, D_{\mathrm{j} . \mathrm{k}}$, and $Z_{\mathrm{j} . \mathrm{k}}$ from HS and VS, when SR and SE are symmetrically deflected are considered. Aerodynamic forces $L_{\mathrm{al}}$ and $D_{\mathrm{al}}$ created by slightly deflectable ailerons may be introduced. Products of each said concentrated and distributed forces multiplied by distances or force arms to the relevant local aerodynamic centers and GC form the static and control moments shown in Figure 2. 


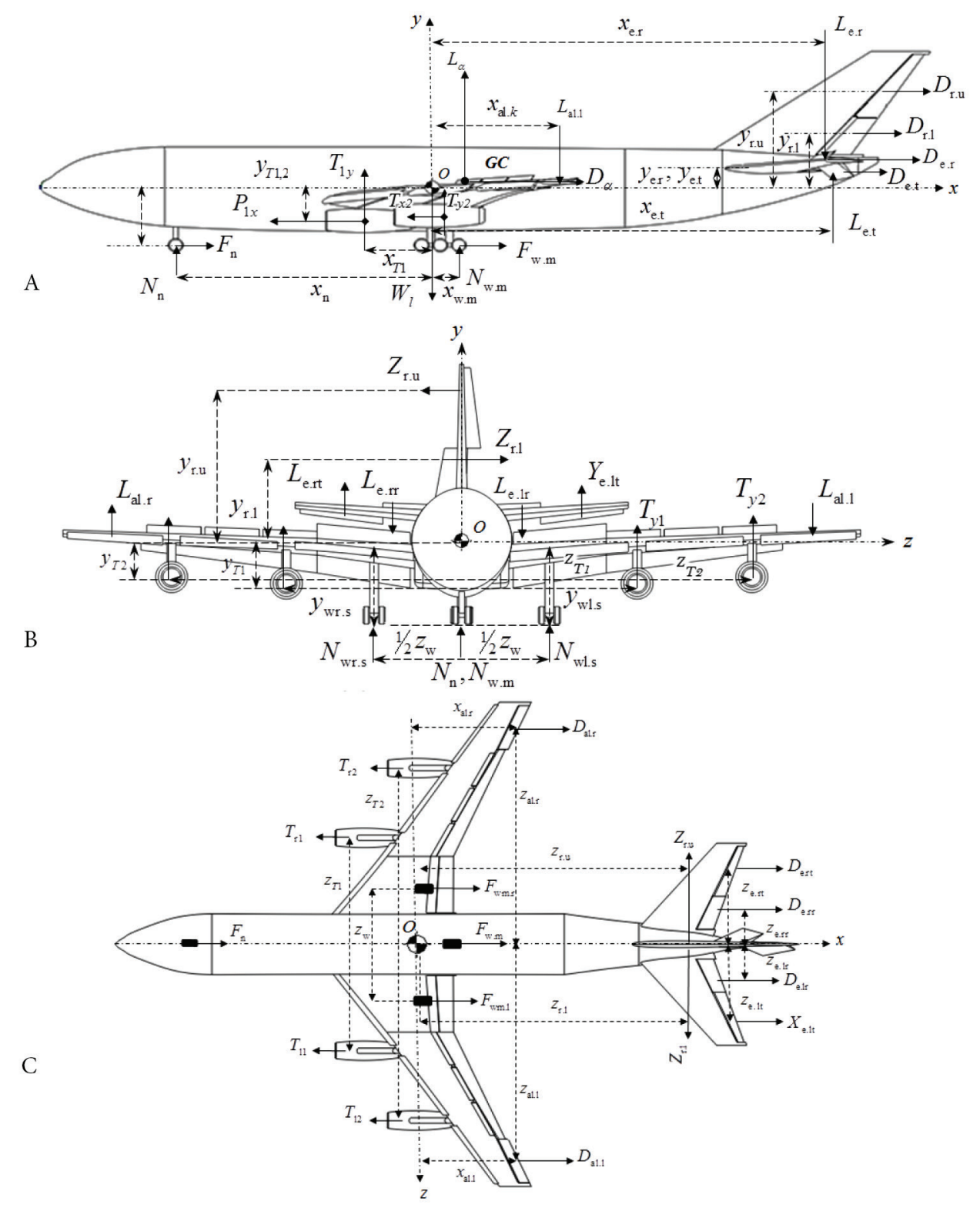

Figure 2. Forces influencing Il-86 during short landing run with symmetrically deflected SR and SE

Consequently, when neglecting deformation of tires and shock-absorber struts at the moment of touchdown body-axis moment equations can be written as sums of moment coefficients projected on $O x y, O x z, O y z$ as depicted in Figure 2:

$$
\begin{aligned}
& \sum m_{R x}=\Delta m_{x}^{\delta_{r}} \delta_{\mathrm{r}}+\Delta m_{x}^{\delta_{\mathrm{e}}} \delta_{\mathrm{e}}+m_{\sum T y}-m_{x}^{\delta_{\mathrm{all}}} \delta_{\mathrm{al}}-\frac{\Delta N}{A q} \bar{z}_{\mathrm{w}} ;(1) \\
& \sum m_{R y}=m_{\sum T x}+m_{y}^{\delta_{n}} \delta_{\mathrm{n}}+\Delta m_{y}^{\delta_{r}} \delta_{\mathrm{r}}+ \\
& \Delta m_{y}^{\delta_{\mathrm{e}}} \delta_{\mathrm{e}}-m_{y}^{\delta_{a l}} \delta_{\mathrm{al}}-m_{y F_{\Delta N}} ; \\
& \sum m_{R z}=m_{z}+m_{\sum T}+m_{z N_{\mathrm{n}}}-m_{z N_{\mathrm{w} . \mathrm{m}}}-m_{z F_{\mathrm{n}}}-m_{z F_{\mathrm{w} . \mathrm{m}}}- \\
& m_{z N_{\mathrm{w} . \mathrm{s}}}-m_{z F_{\mathrm{w} . \mathrm{s}}}+m_{z}^{\delta_{\mathrm{al}}} \delta_{\mathrm{al}}+m_{z}^{\varphi} \varphi+m_{z}^{\delta_{\mathrm{r}}} \delta_{\mathrm{r}}+\Delta m_{z}^{\delta_{\mathrm{e}}} \delta_{\mathrm{e}} .
\end{aligned}
$$

In general, design of sectioned control surfaces provides control system reliability enhancement and longitudinal and directional controllability improvement at cruising speed (Bekhtir, 1991). Area of rudder and elevator usually is split in half to ensure an equal distribution of the spanwise structural loads on each section.

Let us consider system of Eqs (1)-(3) for aircraft of a conventional landing configuration with respect to the described parameters and considerations in assumptions (13). Obviously, $\Delta m_{x}^{\delta_{\mathrm{e}}}=\Delta m_{y}^{\delta_{\mathrm{e}}}=0$ and $m_{\sum T y}=m_{\sum T_{x}}=0$ because of flow and thrust symmetry. Safe landing run stipulates three-point contact with runway, thus $\sum m_{R x}=\sum m_{R z}=0$ must be achieved. Only two degrees of motion freedom are permitted, i.e. forward along $O_{g} x_{g}$ of global coordinate system and yaw around $O y$ body-axis 
coordinate system. Accordingly, Eqs (1)-(3) will be rewritten as follows:

$$
\begin{aligned}
& \Delta m_{x}^{\delta_{\mathrm{r}}} \delta_{\mathrm{r}}-m_{x}^{\delta_{\mathrm{al}}} \delta_{\mathrm{al}}-\frac{\Delta N}{A q} \bar{z}_{\mathrm{w}}=0 \\
& \sum m_{R y}=m_{y}^{\delta_{\mathrm{n}}} \delta_{\mathrm{n}}+\Delta m_{y}^{\delta_{\mathrm{r}}} \delta_{\mathrm{r}}-m_{y}^{\delta_{\mathrm{al}}} \delta_{\mathrm{al}}-m_{y F_{\Delta N}} ;
\end{aligned}
$$

$m_{z}+m_{\sum T}+m_{z N_{\mathrm{n}}}-m_{z N_{\mathrm{w} . \mathrm{m}}}-m_{z F_{\mathrm{n}}}-m_{z F_{\mathrm{w} . \mathrm{m}}}-m_{z N_{\mathrm{w} . \mathrm{s}}}-$

$m_{z F_{\mathrm{w} . s}}+m_{z}^{\delta_{\mathrm{al}}} \delta_{\mathrm{al}}+m_{z}^{\varphi} \varphi+m_{z}^{\delta_{\mathrm{r}}} \delta_{\mathrm{r}}+\Delta m_{z}^{\delta_{\mathrm{e}}} \delta_{\mathrm{e}}=0$.

If $\delta_{\mathrm{al}}=0^{\circ}$ in Eqs (4-5), the created $\Delta m_{x}^{\delta_{\mathrm{r}}}$ by arm length difference of $Z_{r . k}$ contributes an increase of the dynamic loads on the right-side shock-absorber strut pressing more on their tires, which is proportional to deflection angles $\delta_{j . k}$ of SR and SE as seen in Figures 3-4.

Such periodic loadings negatively affect service life of tires. From Figure 2 (A) $\Delta m_{x}^{\delta_{\mathrm{r}}}$ is determined as:

$$
\Delta m_{x}^{\delta_{\mathrm{r}}}=C_{z V S}^{\delta_{\mathrm{r} . \mathrm{u}}} k_{V S} n_{\mathrm{r} . \mathrm{u}} \delta_{\mathrm{r} . \mathrm{u}} \bar{y}_{\mathrm{r} . \mathrm{u}}-C_{z V S}^{\delta_{\mathrm{r} .1}} k_{V S} n_{\mathrm{r} .1} \delta_{\mathrm{r} .1} \bar{y}_{\mathrm{r} .1},
$$

where $k_{V S}=q_{V S} / q$ and $n_{\mathrm{r} . \mathrm{u}}=\sqrt{A_{\mathrm{r} . \mathrm{u}} / A}, n_{\mathrm{r} . \mathrm{l}}=\sqrt{A_{\mathrm{r} . \mathrm{l}} / \mathrm{A}}$ (Mkhitaryan et al., 2012). Also, $n_{\text {r.u }}=n_{\text {r.l }}$ according to the above-mentioned assumption (1). Considering motion with $\alpha_{d}$ and landing speed $V_{l}$ the parameter $k_{V S}$ can be regarded equal along VS. When $\Delta m_{x}^{\delta_{\mathrm{r}}}<0$ resulting in $\Delta N$ a slight leftward yaw may be observed, see Figure 6. For

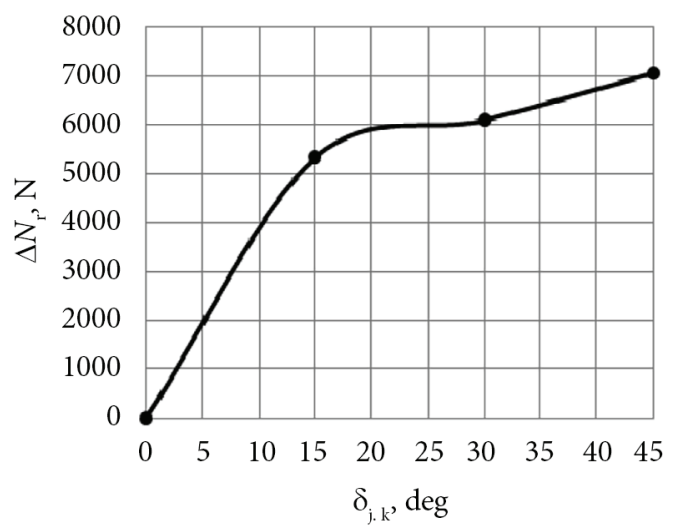

Figure 3. Normal force increase on main landing gear right-side tires

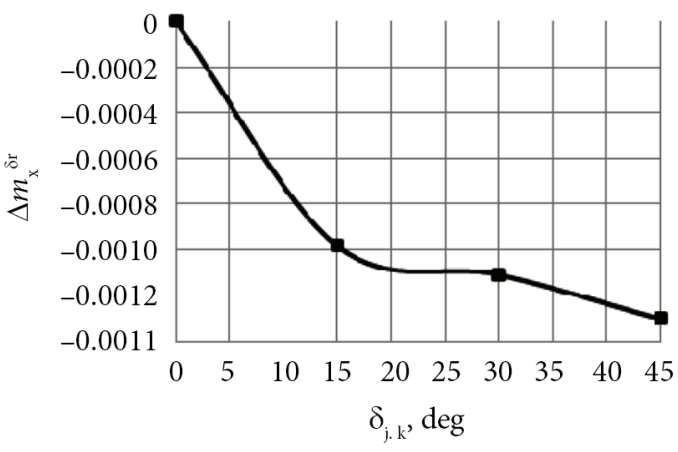

Figure 4. Change of $\Delta m_{x}^{\delta_{r}}$ as function of $\delta_{j . k}$
$\Delta m_{x}^{\delta_{\mathrm{r}}}>0$ one observes the opposite. However, for safe and stable landing run pressure on wheel brakes should not exceed the levels approved by the manufacturer the braking system. In addition, excessive wear of brakes and one or more tires should be always avoided (FAA, 2018).

Full deployment of air-brakes decelerates aircraft and significantly reduces effectiveness of its control surfaces including $S R$ and $S E$ as exemplified in Figure 5. Numerical solution of Eq. (5) reveals the trivial effect of $\Delta m_{x}^{\delta_{r}}$ below $30 \mathrm{~m} / \mathrm{s}$ on the straightness of the landing run. Lowering $V_{l}$ over landing timespan as demonstrated in Figure 5 and small $\Delta x_{\mathrm{r} . k}$ enable offsetting $\Delta m_{x}^{\delta_{\mathrm{r}}}$ using mechanical or aerodynamic approaches.

At speeds near $V_{l}$ ailerons still effective, therefore, they can be set at a trimming angle $\delta_{\mathrm{al}}$. If $m_{\tilde{o}}^{\delta_{\text {al }}}$ realizing $\Delta N=0$ is known, then from Eq. (4) $\delta_{\mathrm{al}}$ is:

$$
\delta_{\mathrm{al}}=\frac{\Delta m_{x}^{\delta_{\mathrm{r}}}}{m_{x}^{\delta_{\mathrm{al}}}} \delta_{\mathrm{r}} .
$$

Analogically, using Eq. (5) one obtains:

$$
\delta_{\mathrm{n}}=\frac{m_{\dot{\alpha}}^{\delta_{\mathrm{al}}} \delta_{\mathrm{al}}-\Delta m_{\dot{o}}^{\delta_{\mathrm{r}}} \delta_{\mathrm{r}}}{m_{y}^{\delta_{\mathrm{n}}}} \text {. }
$$

In general, airplane handbooks allow aileron implementation for directional control in takeoff and landing modes as they still effective if not leading to unpredicted

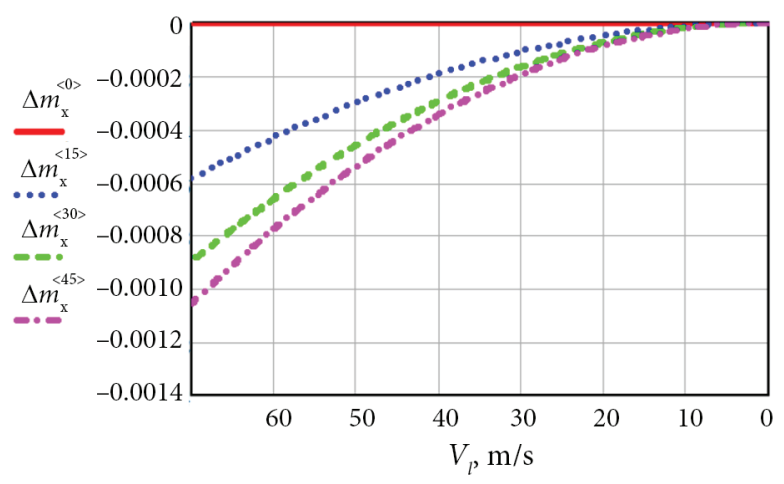

Figure 5. Decrease of $\Delta m_{x}^{\delta_{\mathrm{r}}}$ for each $\delta_{j . k}$ during landing run

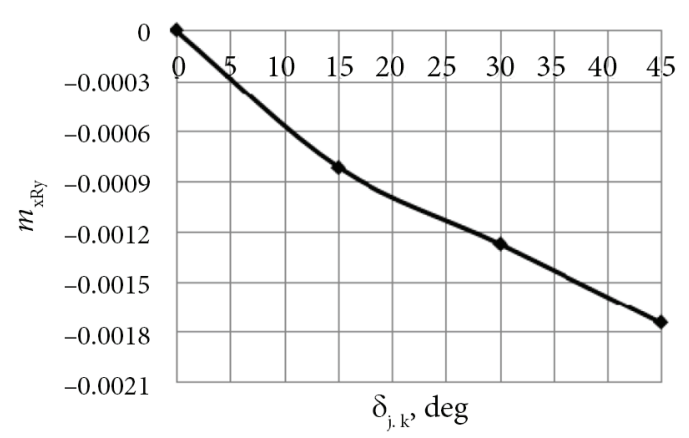

Figure 6. Change of $m_{R y}$ as function of SR and SE deflection at $\delta_{j . k}$ 
consequences. Moreover, transport aircraft are also allowed to use rudder and aileron for landing stabilization during crosswinds (FAA, 2017, 2018). Note that the term $m_{y}^{\delta_{\text {al }}}$ in Eq. (9) also should count the effect of asymmetrical flow around ailerons due to Il-86 wing geometric twist, which approximately is $-3^{\circ}-\left(-4^{\circ}\right)$ at low airspeeds (Bekhtir, 1991). When left aileron is deflected upwards, it creates more aerodynamic forces than the right one that is deflected downwards. Mechanical approach suggests turning front wheel with $\delta_{\mathrm{n}}$ to recover straight-forward landing run. Accordingly, on Table 1 trim angles $\delta_{\mathrm{al}}$ and $\delta_{\mathrm{n}}$ are listed. Note that trimming $\delta_{\mathrm{n}}$ is small, while $\delta_{a l}$ is effective only within a short segment of runway length, where $V_{l}>$ $30 \mathrm{~m} / \mathrm{s}$.

Table 1. Required trim angles $\delta_{\mathrm{al}}$ and $\delta_{\mathrm{n}}$

\begin{tabular}{|c|c|c|c|}
\hline$\delta_{\mathrm{j}}$ & $\delta_{\mathrm{j} . \mathrm{k}}=15^{\mathrm{o}}$ & $\delta_{\mathrm{j} . \mathrm{k}}=30^{\mathrm{o}}$ & $\delta_{\mathrm{j} . \mathrm{k}}=45^{\mathrm{o}}$ \\
\hline$\delta_{\mathrm{al}}$ & $-4.9^{\mathrm{o}}$ & $-5.6^{\mathrm{o}}$ & $-6.6^{\mathrm{o}}$ \\
\hline$\delta_{\mathrm{n}}$ & $-1^{\mathrm{o}}$ & $-0.53^{\mathrm{o}}$ & $-0.73^{\mathrm{o}}$ \\
\hline
\end{tabular}

In scenarios where it is allowed, pilot may use differential braking to maintain directional control (FAA, 2018). This is implied by solving Eq. (5) with $\delta_{\mathrm{al}}=0^{\circ}$ resulting in $\Delta m_{y}^{\delta_{\mathrm{r}}}=m_{y F_{\Delta N}}-m_{y}^{\delta_{\mathrm{n}}} \delta_{\mathrm{n}}$, whereas for trimming $\Delta m_{y}^{\delta_{\mathrm{r}}}$ differential braking is expressed as follows (Buchkarev et. al, 1985):

$$
m_{y F_{\Delta N}}=-\frac{\Delta N_{\text {w.s }} \bar{z}_{\mathrm{w}} \mu}{2 q A} .
$$

Both operational approaches overload pilots with extra physiological stress, especially for aircraft with high $V_{l}$. One of the key constraints on the landing process is that it should not require exceptional skills or excessive force from the pilot (FAA, 1997). Simultaneous or successive switching of lift-dampers, spoilers, flaps and slats along with braking and nose wheel steering and thrust reversers may distract and overload pilot threatening safety of landing (FAA, 2018). Therefore, modern aircraft are equipped with automatic braking and AHLCS integrated with the automatic landing program. Further modification aimed to prevent extreme deviations of landing parameters can remove significantly the possible extra stress during manual piloting.

Operational approaches depend on dynamic balancing measurements. However, they are replaceable by built-in angle deflection limitations introduced at early design stages of the flight control system. Deflection angles $\delta_{\text {r.u }}$ may be structurally constrained for ensuring $\delta_{\text {r.u }} \neq \delta_{\text {r.l }} \Rightarrow \Delta m_{x}^{\delta_{\mathrm{r}}}=0$. Thus, for achieving $\Delta N=0$ at $\delta_{\mathrm{al}}=0^{\circ}$ Eq. (4) suggests inherent trim angle $\delta_{\text {r.u }}^{\prime}$ as:

$$
\delta_{\text {r.u }}^{\prime}=\frac{C_{z \text { r.l. }}^{\delta_{\text {r. }}} \bar{y}_{\text {r.l }}}{C_{z \text { r.u }}^{\delta_{\text {r.u }}} \bar{y}_{\text {r.u }}} \delta_{\text {r.l }} .
$$

Unlike operational approaches, setting SR at $\delta_{\text {r.u }}^{\prime}$ reduces air-braking efficiency manifesting a disadvantage of this approach. In addition, complete elimination of the potential extra physiological stress may remain unachieved since $\Delta m_{x}^{\delta_{\mathrm{r}}} \neq \Delta m_{y}^{\delta_{\mathrm{r}}}$ as $\Delta x_{r . k} \neq \Delta y_{r . k}$. Moreover, the required re-design of travel limits of the SR with respect to $\delta_{\text {r.u }}^{\prime}$ decreases its maximum angles and complicates modification process of the existing aircraft. Therefore, achieving $\delta_{\text {r.u }}^{\prime}$ is safer and more feasible using programmed or manual differential deflection of SR sections to opposite unequal angles. Note that the problem of $\Delta m_{x}^{\delta_{\mathrm{r}}}$ characterizes only tail sections with an odd number of fins. As for the cargo airplane An-225 with two fins and two SR, deflection of both SR results in $\Delta m_{x V S}^{\delta_{\mathrm{r}}}=0$, since $\delta_{\text {r.u }}$ and $\delta_{\text {r.l }}$ at each fin deflect oppositely.

On the other hand, from Eq. (6) one notices additional pitching moments around $\mathrm{O} z$ caused by deflecting SR and SE. Symmetrical deflection of SR always creates some positive pitching moment $m_{z}^{\delta_{\mathrm{r}}}>0$, which increases by headwind:

$$
m_{z}^{\delta_{\mathrm{r}}}=C_{x V S}^{\delta_{\mathrm{ru}}} k_{V S} n_{\mathrm{r} . \mathrm{u}} \delta_{\mathrm{r} . \mathrm{u}} \bar{y}_{\mathrm{r} . \mathrm{u}}+C_{x V S}^{\delta_{\mathrm{r} .1}} k_{V S} n_{\mathrm{r} . \mathrm{l}} \delta_{\mathrm{r} . \mathrm{l}} \bar{y}_{\mathrm{r} . \mathrm{l}} .
$$

In fact, effects of SE symmetrical deflection on the total pitching moment are complex. For sweptback HS, difference between distances to GC from local aerodynamic centers on deflected surfaces, i.e. $x_{\text {e.t }}$ and $y_{\text {e.t }}$ are a bit farther than $x_{\text {e.r }}$ and $y_{\text {e.r }}$. This creates $\Delta m_{z}^{\delta_{e}}$, which increases as $\chi_{S t}$ diverges. In result, there are two cases: $\Delta m_{z}^{\delta_{\mathrm{e}}}<0$ or $\Delta m_{z}^{\delta_{\mathrm{e}}}>0$ that depend on $\delta_{\mathrm{e}}$ and $\varphi$. Additionally, aircraft motion in the proximity of the ground surface with landing configuration is influenced by flow interference, namely by the considerable vertical and horizontal flow drifting in the zone behind the wing-trailing edge and around HS as captured in Figure 7(A) and (B).

Total moment coefficient around $\mathrm{O} z$ axis $\left(m_{R z}\right)$ of Il-86 with full landing configuration and relevant aerodynamic phenomena as flow drifting may produce $m_{R z}<0$ complicating touchdown on main wheels or $m_{R z}>0$ increasing the probability of a tailstrike at touchdown. Avoiding such consequences is achievable by deflecting SR and SE exclusively after touchdown simultaneously with thrust reversing. For Il-86 with center-of-gravity position 16-33\% of MAC (Bekhtir, 1991) $m_{\Sigma T}=-0.038$ during thrust reversal, $m_{z}^{\delta_{\mathrm{r}}}>0$ and $m_{z H S}>0$ all result in $m_{R z}>0$ as seen in Figures 8-9. However, pitching moments generated by friction, normal, and drag forces from landing gear wheels and struts trim $m_{R z}$. In general, while taxing $80-85 \%$ of $W_{l}$ is borne by main landing gear leaving 20-15\% for nose landing gear (Mkhitaryan et al., 2012). This distribution may vary depending on landing conditions. For instance, when SE are symmetrically deflected according to $\delta_{\text {e.kt }}=-\delta_{\text {e.kr }} \Delta m_{z}^{\delta_{\mathrm{e}}}<0$ is noticed. It mostly and unfavorably influences $m_{z N_{\mathrm{n}}}$ and $m_{z F_{\mathrm{n}}}$ as $F_{\mathrm{n}}$ magnitude increase, which sometimes becomes worse during rough landings or tailwind.

Maintaining the desired longitudinal balancing, i.e. $\Delta m_{z}^{\delta_{\mathrm{e}}}=0$, for aircraft with fixed stabilizer is achievable by 


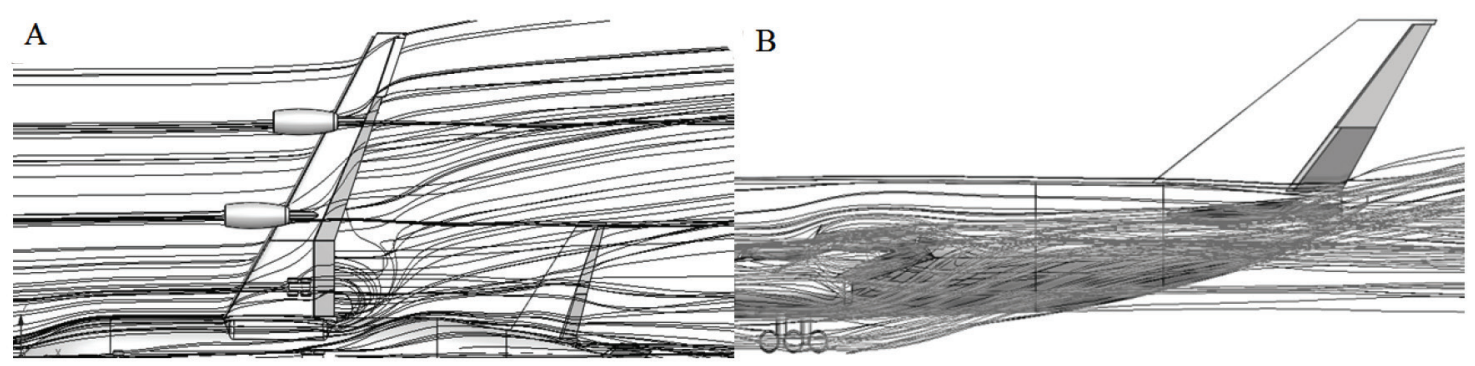

Figure 7. Flow streamlines and interference behind the wing-tailing edge at $V_{l}=77.87 \mathrm{~m} / \mathrm{s}$ viewed from: A - upper view on $O x z$ plane showing horizontal flow drifting; B - side view on Oxy plane showing vertical flow drifting

applying the same technique suggested in Eq. (11). Generally, $\Delta m_{z}^{\delta_{e}}$ is given as:

$$
\begin{aligned}
& \Delta m_{z}^{\delta_{e}}=k_{\text {e.t }} n_{\text {e.t }} \delta_{\text {e.t }}\left(C_{y e . t}^{\delta_{\text {e.t }}} \bar{x}_{\text {e.t }}+C_{x e . t}^{\delta_{\text {e.t }}} \bar{y}_{\text {e.t }}\right)- \\
& k_{\text {e.r }} n_{\text {e.r }} \delta_{\text {e.r }}\left(C_{y e . r}^{\delta_{e . r}} \bar{x}_{\text {e.r }}+C_{x e . r}^{\delta_{\text {e.r }}} \bar{y}_{\text {e.r }}\right) .
\end{aligned}
$$

Here, $n_{\text {e.t }}=n_{\text {e.r }}$ and $k_{\text {e.t }}>k_{\text {e.r }}$ because of flow interference (Figure 7). However, this relation becomes $k_{\text {e.t }}<k_{\text {e.r }}$ in the second deflection option of SE $\left(\delta_{\mathrm{e} . k \mathrm{r}}=-\delta_{\mathrm{e} . k \mathrm{t}}\right)$. Here, $\Delta m_{z}^{\delta_{\hat{\mathrm{a}}}}<0$ is observed, thus $N_{\mathrm{n}}$ and $F_{\mathrm{n}}$ increase. Derivation of $\delta_{\text {e.t }}^{\prime}$ from Eq. (13) produces:

$$
\delta_{\text {e.t }}^{\prime}=\frac{k_{\text {e.r }} n_{\text {e.r }}\left(C_{y e . r}^{\delta_{\text {e.r }}} \bar{x}_{\text {e.r }}+C_{x e . r}^{\delta_{\text {e.r }}} \bar{y}_{\text {e.r }}\right)}{k_{\text {e.t }} n_{\text {e.t }}\left(C_{y e . t}^{\delta_{\text {e.t }}} \bar{x}_{\text {e.t }}+C_{\text {e.t. }}^{\delta_{\text {e.t }}} \bar{y}_{\text {e.t }}\right)} \delta_{\text {e.r }}
$$

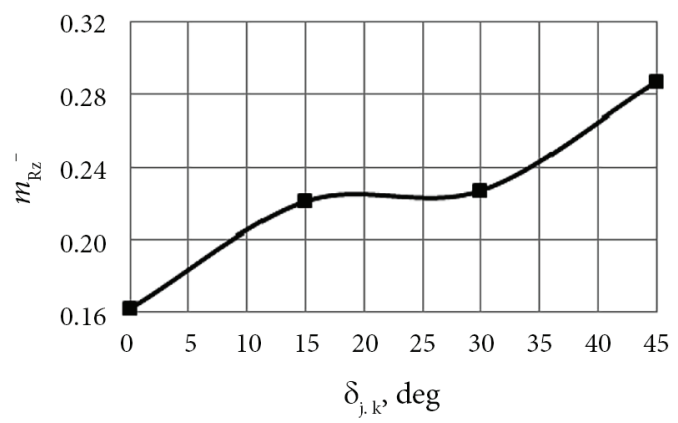

Figure 8. Change of $m_{R z}$ as function of $\delta_{j . k}$

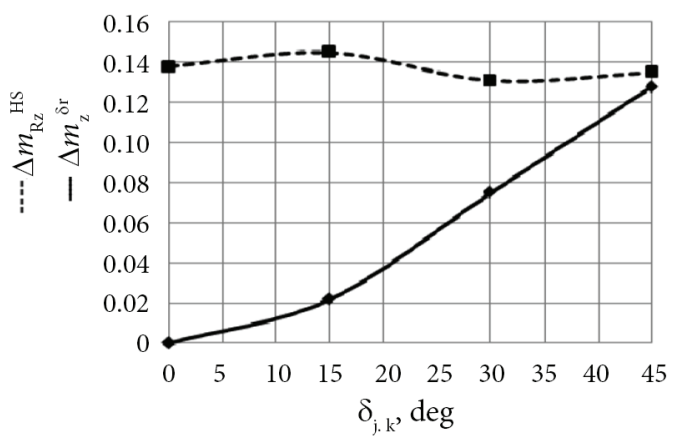

Figure 9. Change of $m_{z H S}$ and $m_{z}^{\delta_{\mathrm{r}}}$ as function of $\delta_{j . k}$
Similarly, such a limited $\delta_{\text {e.t }}^{\prime}$ reduces SE air-braking efficiency, which should be avoided.

For aircraft with adjustable-incidence tailplane an alternative method consisting in deflecting HS to some $\varphi_{\operatorname{tr}}$ can be implemented. Such an approach trims the additional moments $\Delta m_{z}^{\delta_{\mathrm{e}}}, \Delta m_{z}^{\delta_{\mathrm{al}}}$, and $\Delta m_{z}^{\delta_{\mathrm{r}}}$, while providing good margin of longitudinal stability to prevent nosewheel lift-off especially during relatively strong headwind. Using Eq. (6) Table 2 contains the required $\varphi_{\text {tr }}$ for Il-86:

Table 2. Trim angles for adjustable-incidence tailplane of Il-86 at $V_{l}=77.87 \mathrm{~m} / \mathrm{s}$

\begin{tabular}{|c|c|c|c|}
\hline & $\delta_{j . k}=15^{\circ}$ & $\delta_{j . k}=30^{\circ}$ & $\delta_{j . k}=45^{\circ}$ \\
\hline$\varphi_{\operatorname{tr}}$ & $-1.5^{\circ}$ & $-1.7^{\circ}$ & $-2.1^{\circ}$ \\
\hline
\end{tabular}

Consequently, this enables avoiding implementing $\delta_{\text {e.t }}^{\prime}$ and simplifies necessary modifications in the longitudinal stability control system. Formula of $\varphi_{\text {tr }}$ can be written in an expanded form as follows:

$$
\begin{aligned}
& \varphi_{\mathrm{tr}}=\frac{-m_{z 0}^{H L}-\left(\bar{x}_{C G}-\bar{x}_{F c}\right)\left(C_{L \alpha}+\Delta C_{L \alpha \mathrm{gr}}\right)-}{m_{z}^{\varphi_{\mathrm{tr}}}} \\
& \frac{\left(\bar{x}_{\mathrm{CG}}-\bar{x}_{F 2}\right) \Delta C_{L a}^{H L}-m_{z 0}^{H L}-\Delta m_{z}^{\delta_{\mathrm{e}}} \delta_{\mathrm{e}}-\Delta m_{z}^{\delta_{\mathrm{al}}} \delta_{\mathrm{al}}-\Delta m_{z}^{\delta_{\mathrm{r}}} \delta_{\mathrm{r}}}{m_{z}^{\varphi_{\mathrm{tr}}}} .
\end{aligned}
$$

For tailless aircraft such as Concorde and Tu-144, as well as "flying wing" configuration such as B-2 flow drifting is zero. For Tu-144 $\chi_{\mathrm{st}}=0^{\circ}$ leading to $\Delta m_{z}^{\delta_{\mathrm{e}}}=0$.

\section{Conclusions}

With the aim of addressing remarks outlined in a previous numerical study concerning few landing run phase instabilities emerging after the deflection of tail part split control surfaces for shortening landing run of Il-86 aircraft this work detailed a number of operational and mechanical approaches proposed to maintain aircraft balance during a straight-line landing run under standard weather and runway conditions. Mechanical approach is disadvantageous and less competent because it requires re-design 
of control surfaces attachments to include structural limiters of deflection angles. In contrary, operational approaches comprise of less system architecture complexity and suggest manual or automatic differential braking, nose wheel steering, aileron, and/or adjustable-incidence tailplane setting at specific trimming angles to offset the untrimmed small pitching, yawing, and rolling moments peaking directly after touchdown. The quick deceleration during the landing run causes rapid attenuation of these moments until reaching $30 \mathrm{~m} / \mathrm{s}$ where they become insignificant. Up-to-date automatic high-lift and braking systems incorporated in modern aircraft control programs provide the basis for successful modification of sectioned rudder and elevator control algorithms to qualify for safe and effective application as air-brakes without overloading pilot or requiring his exceptional skills.

\section{Disclosure statement}

The author declares that he has no financial, professional, or personal interests from other parties that relate to the research described in this paper.

\section{References}

Alyamovsky, A. A. (2012). SolidWorks Simulation, Kak reshat prakticheskie zadachi [SolidWorks Simulation. How to solve practical problems]. Saint Petersburg: Saint Petersburg Publisher.

Ba Zuhair, M. A. (2018). R.U. Patent No. 2,668,000. Moscow: R.U. Patent and Trademark Office.

Bazuhair, M. A. (2018). A technique for shortening landing run distance of an aircraft by symmetrical deflection of split elevator and rudder. Russian Aeronautics, 61(2), 187-193. https://doi.org/10.3103/S106879981802006X

Bekhtir, V. P. (1991). Prakticheskaja ajerodinamika samoleta Il-86 [Il-86 aircraft performance]. Ulyanovsk: Center of Civil Aviation and Council for Mutual Economic Assistance.

Buchkarev, A., et al. (1985). Aeromechanika samoleta [Aircraft aeromechanics]. Moscow: Mashinostroenie.

Dassualt-Systems. (2015). Flow Simulation 2016 Online user's guide. SOLIDWORKS-2016. Paris: Dassualt Systems. Retrieved from https://www.faa.gov/documentLibrary/media/ Advisory_Circular/AC_25-7D.pdf

Federal Aviation Administration. (2017). Approaches and landings (Chapter 8). In Airplane Flying Handbook, FAA-H-80833B. Retrieved from https://www.faa.gov/regulations_policies/ handbooks_manuals/aviation/airplane_handbook/media/10_ afh_ch8.pdf

Federal Aviation Administration. (2018). Circular 25-7D- Flight Test Guide for Certification of Transport Category Airplanes. Retrieved from https://www.faa.gov/documentLibrary/media/Advisory_Circular/AC_25-7D.pdf

Federal Aviation Administration. (1997). Rules and Regulations: Sino Swearingen Model SJ30-2 Airplane. Retrieved from https://www.govinfo.gov/content/pkg/FR-1997-10-31/pdf/9728937.pdf

Jung, U. S. (2012). Alternative air brake concepts for transport aircraft steep approach (PhD Thesis). Munich Technical University, Munich, Germany.
Liebeck, R. H. (2004). Design of the blended wing body subsonic transport. Journal of Aircraft, 41(1), 10-25. https://doi.org/10.2514/1.9084

Mertol, B. A. (2008). Patent application WIPO/2008/151760. Lifting wing with adjustable spoiler.

Mkhitaryan, A. M., et al. (2012). Dinamika poleta [Eng. trans. Flight Mechanics]. Moscow: EKOLIT publisher.

NASA. (2007). Landing the space shuttle orbiter. NASA. Retrieved from http://www.nasa.gov/pdf/167415main_LandingatKSC-08.pdf

\section{Appendix}

\section{Notations}

\section{Variables and functions}

Re - Reynold's number;

$\alpha$ - angle of attack;

$C_{D \alpha}-$ drag coefficient at given $\alpha$;

$C_{L \alpha}-$ lift coefficient at given $\alpha$;

$\alpha_{d}$ - wing design angle of attack;

$V_{l}$ - landing airspeed;

$L_{\alpha}-$ lift at given $\alpha$;

$D_{\alpha}-$ drag at given $\alpha$;

$W_{l}$ - landing weight of aircraft;

$T_{1 j}$ - thrust from pair engines 1 ;

$T_{2 j}$ - thrust from pair engines 2 ;

$F_{n}$ - friction force from nose wheel;

$F_{\text {r.k }}$ - friction force from " $\mathrm{k}$ " nose wheel;

$N_{\mathrm{n}}$ - normal force from nose wheel;

$N_{\text {r.k }}$ - normal force from "k" rare wheel;

$L_{\mathrm{j} . \mathrm{k}}-$ lift from slightly deflected at " $\mathrm{j} . \mathrm{k}$ ";

$D_{\mathrm{j} . \mathrm{k}}-$ drag from slightly deflected at "j.k";

$Z_{\mathrm{j} . \mathrm{k}}$ - lateral force from slightly deflected at " $\mathrm{j} . \mathrm{k}$ ";

$L_{\mathrm{al}}$ - lift slightly deflectable ailerons;

$D_{\mathrm{al}}$ - drag from slightly deflectable ailerons;

$\delta_{\mathrm{r}}$ - deflection angle of SR sections;

$\Delta m_{x}^{\delta_{\mathrm{r}}}-$ partial derivative of rolling moment coefficient

as function of $\delta_{r}$;

$\delta_{\mathrm{e}}$ - deflection angle of SE sections;

$\Delta m_{x}^{\delta_{\mathrm{e}}}$ - partial derivative of rolling moment coefficient as function of $\delta_{\mathrm{e}}$;

$m_{\sum T y}$ - rolling moment coefficient from the vertical component of thrust by jet engines 1 and 2;

$\delta_{\mathrm{al}}$ - angle of aileron deflection;

$m_{x}^{\delta_{a l}}$ - rolling moment coefficient, when ailerons are deflected by $\delta_{\mathrm{al}}$;

$\Delta N$ - change of normal forces on the main landing gear right or left wheels due to rolling moment;

$z_{\mathrm{w}}$ - distance between the main landing gear wheel tracks $z_{\mathrm{w}}$; 
$\bar{z}_{\mathrm{w}}$ - relative distance between the main landing gear wheel tracks;

$d$ - wingspan;

$q$ - flow dynamic pressure;

$A$ - reference area of the wing;

$m_{\sum T_{x}}$ - yawing moment coefficient from the horizontal component of thrust by engines 1 and 2;

$\delta_{\mathrm{n}}$ - nose wheel rotation angle;

$m_{y}^{\delta_{\mathrm{n}}}$ - yawing moment coefficient of nose wheel;

$\delta_{\mathrm{r}}$ - deflection angle of rudder;

$\delta_{\mathrm{e}}-$ deflection angle of elevator;

$\Delta m_{y}^{\delta_{\mathrm{r}}}$ - partial derivative of yawing moment coefficient as function of $\delta_{\mathrm{r}}$;

$\Delta m_{y}^{\delta_{\mathrm{e}}}$ - partial derivative of yawing moment coefficient as function of $\delta_{\mathrm{e}}$;

$m_{o}^{\delta_{\text {al }}}$ - yawing moment coefficient, when ailerons are deflected by $\delta_{\mathrm{al}}$;

$m_{y F_{\Delta N}}$ - yawing moment coefficient due to $\Delta \mathrm{N}$;

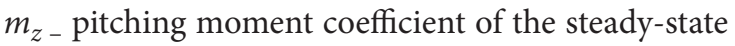
trimmed flight;

$m_{\sum T}$ - pitching moment coefficient from thrust;

$m_{z N_{\mathrm{n}}}$ - pitching moment coefficient from normal force created by nose wheels;

$m_{z N_{\mathrm{w} . \mathrm{m}}}$ - pitching moment coefficient from normal force created by middle wheels;

$m_{z N_{\text {w.s }}}$ - pitching moment coefficient from normal force created by side (left and right) wheels;

$m_{z F_{\mathrm{n}}}$ - pitching moment coefficient from friction force created by nose wheels;

$m_{z F_{\mathrm{w} . \mathrm{m}}}$ - pitching moment coefficient from friction force created by middle wheels;

$m_{z F_{\text {w.s }}}$ - pitching moment coefficient from friction force created by side (left and right) wheels;

$m_{z}^{\delta_{\text {al }}}$ - pitching moment coefficients, when ailerons are deflected by $\delta_{\mathrm{al}}$;

$\varphi$ - incidence angle of HS;

$m_{z}^{\varphi}$ - pitching moment coefficient influenced by incidence angle of HS $(\varphi)$;

$m_{z}^{\delta_{\mathrm{r}}}$ - pitching moment coefficient, when $\delta_{\mathrm{r}}>0$;

$\Delta m_{z}^{\delta_{\mathrm{e}}}$ - partial derivative of pitching moment coefficient as a function of $\delta_{\mathrm{e}}$;

$C_{z V S}^{\delta_{\text {r.u }}}-$ derivative term of VS lateral force at setting angle $\delta_{\text {r.u }}$;

$C_{z V S}^{\delta_{\text {r.l }}}$ - derivative term of VS lateral force at setting angle $\delta_{\text {r.l }}$;

$k_{V S}$ - coefficient of the flow deceleration around VS;
$q_{V S}$ - dynamic pressure around VS;

$n_{\text {r.k }}$ - relative effectiveness coefficients of each " $k$ " SR section;

$\mu$ - friction coefficient;

$C_{x V S}^{\delta_{\text {r.u }}}$ - derivative term of VS drag at $\delta_{\text {r.u }}>0$;

$C_{x V S}^{\delta_{\text {r.l }}}-$ derivative term of VS drag at $\delta_{\text {r.l }}>0$;

$x_{\text {e.t }}, x_{\text {e.r }}$ - horizontal distance to local aerodynamic center of elevator tip ".t" or root ".r" sections;

$y_{\text {e.t }}, y_{\text {e.r }}$ - vertical distance to local aerodynamic center of elevator tip ".t" or root ".r" sections;

$\Delta x_{\text {r. } k}$ - difference between horizontal distances from $\mathrm{GC}$ to local aerodynamic center of rudder tip ".t" or root ".r" sections;

$\chi_{S t}$ - stabilizer sweepback angle;

$C_{L \text { e.t }}^{\delta_{\text {e.t }}}$ - derivative term of SE lift coefficient at given $\delta_{\text {e.t }}$;

$C_{\text {Le.r }}^{\delta_{\text {e.r }}}$ - derivative term of SE lift coefficient at given $\delta_{\text {e.r }}$;

$C_{\text {De.t }}^{\delta_{\text {e.t }}}-$ derivative term of SE drag coefficient at given $\delta_{\text {e.t }}$;

$C_{D e . r}^{\delta_{e . r}}$ - derivative term of SE drag coefficient at given $\delta_{\text {e.r }}$;

$m_{z 0}^{H L}$ - pitching moment coefficient, when

$C_{L a}=\delta_{j . k}=\varphi=0$;

$\bar{x}_{\mathrm{CG}}$ - relative center-of-gravity position;

$\bar{x}_{F c}-$ zero-a pitching moment coefficient from engines;

$\Delta C_{\text {Lagr }}$ - incremental lift coefficient caused by ground effect;

$\bar{x}_{F 2}$ - relative center of incremental lift caused by full deployment of high-lift devices, or so-called "second" focus;

$\Delta C_{L a}^{H L}$ - incremental lift coefficient caused by full deployment of high-lift devices;

$\varphi_{\mathrm{tr}}$ - deflection angle of adjustable-incidence tailplane; $m_{z}^{\varphi_{\text {tr }}}-$ pitching moment coefficient at $\varphi_{\mathrm{tr}}$.

\section{Abbreviations}

HS - horizontal stabilizer;

VS - vertical stabilizer;

CFD - computational fluid dynamics;

RANS - Reynolds Averaged Navier-Stokes;

SR - sections of rudder;

SE - sections of elevator;

GC - center of gravity;

AHLCS - automatic high-lift control system;

MAC - main aerodynamic chord. 